\title{
CDISC SDTM Pharmacogenomics Findings Test Code Terminology
}

National Cancer Institute

\section{Source}

National Cancer Institute. CDISC SDTM Pharmacogenomics Findings Test Code

Terminology. NCI Thesaurus. Code C116106.

Terminology associated with the pharmacogenomics findings test code codelist of the Clinical Data Interchange Standards Consortium (CDISC) Study Data Tabulation Model (SDTM). 